DOI 10. 18307/2016. 0201

(C) 2016 by Journal of Lake Sciences

\title{
非传统湖泊水色遥感的现状与发展
}

\author{
马荣华,张玉超,段洪涛 \\ ( 中国科学院南京地理与湖泊研究所湖泊与环境国家重点实验室,南京 210008)
}

摘 要: 外界环境条件以及自身因素的驱动,改变了传统湖泊水色遥感垂向均一的理论假设前提,基于垂向非均匀条件 的湖泊水体水色参数的遥感称之为非传统湖泊水色遥感. 本文分析了传统水色遥感面临的挑战, 且非传统湖泊水色遥感 中藻类叶绿素 $\mathrm{a}$ 浓度的垂向分布类型及其定量表达、水下光场分布的定量表达模型与数值模拟方法, 给出垂向异质水体 遥感反射比的定量表达式, 分析了藻类垂向异质对水色参数遥感反演模型的影响, 最后提出下一步需要重点关注的问题.

关键词: 水色遥感;垂向;非传统; 现状;发展;湖泊

\section{The status and development of the non-traditional lake water color remote sensing}

MA Ronghua, ZHANG Yuchao \& DUAN Hongtao

( State Key Laboratory of Lake Science and Environment, Nanjing Institute of Geography and Limnology, Chinese Academy of Sciences, Nanjing 210008, P.R.China)

\begin{abstract}
We define "non-traditional water color remote sensing” as the study of the heterogeneous vertical distribution of water color parameters and their inherent optical properties, in relation to the environmental factors (external and internal) that influence their distribution. This is quite different from traditional water color remote sensing as it includes the third dimension, rather than assuming homogeneous vertical distribution of water color parameters. In this study, we explore approaches to determine the vertical distribution of chlorophyll-a and explore methods to simulate the underwater light field. It should be noted that remote sensing reflectance $\left(R_{\mathrm{rs}}\right)$ of non-uniform water column could be equivalent to optically weighted $R_{\mathrm{rs}}$ with vertical uniform distribution. The effects of non-uniform vertical profiles of chlorophyll-a concentration on inversion models of remote-sensing is explored, and challenges should be the focus of further research.
\end{abstract}

Keywords: Water color remote sensing; vertical distribution; non-traditional; status; development; lake

在湖泊水体水色三要素参数的定量反演方面, 已经进行了大量工作 ${ }^{[1]}$, 并取得了显著成果, 然而, 这些 研究有一个基本的前提假设: 这些水色参数或水体光学特性在垂向上是均质的. 基于该假设的湖泊水体水 色参数的遥感称之为传统的湖泊水色遥感. 实际上, 一方面, 由于河流和冰川等地表径流的汇人、江水倒灌 和风浪等自然驱动力的介人以及采砂、航运、路桥基建等人为活动的扰动, 湖泊底泥再悬浮, 使得湖泊水体 中的悬浮泥沙在垂直方向上非均匀分布; 另一方面, 尤其在富营养化湖泊水体中, 不论在水平方向还是在垂 直方向上, 都存在大量的藻颗粒, 为了保持较高的光合作用能力, 这些藻颗粒通过自身移动或者通过水流和 风生流等外界条件, 不断调节其在水体中的垂直位置, 以躲避强光造成的光抑制或者上浮到水体表层接受 充足的光照形成藻华 ${ }^{[2-7]}$. 这些外环境驱动力以及浮游藻类的主动调节, 客观上造成了藻颗粒及其相关水色 参数 (如叶绿素、藻蓝素、有色可溶性有机物 (colored dissolved organic matter, CDOM) 悬浮物等) 在垂向上的 不均匀分布, 改变了传统湖泊水色遥感的理论假设前提; 这种基于垂向非均匀条件的湖泊水体水色参数的 遥感称为非传统湖泊水色遥感.

\section{1 传统湖泊水色遥感面临的挑战}

湖泊水色参数的垂向非均匀分布, 对于基于传统垂向均一假设而形成的水体光学特性、水下光场分布、

* 国家自然科学重点基金项目(41431176) 资助. 2015-04-20 收稿;2015-06-08 收修改稿. 马荣华( 1972 ), 男, 博士,研究员; E-mail:rhma@ niglas.ac.cn. 
水质参数反演模型等方面都产生了一些难以回避的问题. 传感器获得的遥感信号是水体内一定深度的综 合反映,当假设水体垂向均一时,垂向异质引起的遥感信息的差异,对已有水色遥感的研究造成了不能忽视 的误差. 首先, 湖泊水体内水色物质 (叶绿素 a (Chl. a)、CDOM、悬浮物等) 垂向非均匀分布,会产生相应的 水体固有光学特性垂向异质. 此时, 垂向均一的假设 (某一层或水柱内的平均值代表整个水柱的 IOP (Inherent Optical Properties)) 会引起水柱内固有光学特性的估计不准, 导致以 IOP 为核心的模型, 如 QAA (Quasi-Analytical Algorithm), 直接应用于垂向差异较大的湖泊水体时, 会产生很大误差. 其次, 只有考虑了 水体内固有光学量的垂向异质, 根据辐射传输理论, 才能得到准确的水下光场分布及表观光学量, 否则会引 起估计不准的情况. 在水质参数反演模型的建立及应用方面, 一般是基于垂向均一的假设, 建立光活性物 质浓度与遥感反射比的关系, 而忽略了光活性物质浓度垂向异质对遥感反射比 (Remote Sensing Reflectance, $\left.R_{\mathrm{rs}}\right)$ 的影响, 从而导致定量反演模型在应用中的误差. 实际情况下, 不同物质含量及物质的不同垂向结构, 对 $R_{\mathrm{rs}}$ 的影响程度和范围均不一致, 可能产生反演结果在每个像元的不确定性. 针对传统湖泊水色遥感的垂 向均一假设可能引起的问题及面临的挑战,有必要开展光活性物质垂向异质的研究.

\section{2 藻类叶绿素浓度的垂向分布类型及其定量表达}

目前, 研究水体中藻类垂向分布对水体光学属性的影响, 主要集中在水深较深的大洋水体, 受藻类生活 习性以及外环境条件影响, 藻类垂向分布呈现多种类型, 主要有: 高斯分布 ${ }^{[8]} 、$ 指数分布 ${ }^{[9]}$ 、跃变型、单峰型、 递增型、垂直均匀型、线性分布 ${ }^{[10-11]}$, 其中高斯模型 ${ }^{[8]}$ 在大洋 I 类水体中应用最为广泛 ${ }^{[12-13]}$ :

$$
\operatorname{Chl}(z)=\operatorname{Chl}_{0}+\frac{h}{\delta \cdot \sqrt{2 \pi}} \exp \left[-\frac{1}{2}\left(\frac{z-z_{\text {max }}}{\delta}\right)^{2}\right]
$$

式中, $C h l_{0}$ 为背景叶绿素浓度, 也称为 “本底值”; $\delta$ 为标准偏差, 是与分层中叶绿素极大值宽度有关的参数; $h$ 为与峰值强度有关的参数; $z_{\text {max }}$ 为叶绿素浓度极大值所处的深度. 基于该函数, 国内外学者 ${ }^{[12-14]}$ 评估不同的 水体垂向结构对蓝绿光遥感反射比、离水辐亮度、衰减系数及平均余弦等光学参数影响. 目前, 国内外一些 学者利用统计回归方法 ${ }^{[15-16]}$ 、人工神经网络方法 ${ }^{[17-18]}$ 研究藻类垂向呈高斯分布时对遥感反射比的影响, 同 时可以得到叶绿素浓度垂向呈高斯分布时的参数. 在海洋二流辐射传输理论的基础上 ${ }^{[19]}$, 假设 I 类水体只 由 2 层叶绿素浓度不同的水层构成, 利用最优化方法求得 2 层叶绿素浓度和上层水体深度的最优解. 同样 地, 在红-近红外波段, 遥感反射比与悬浮颗粒物 SPM 2 层结构参数也存在定量关系 ${ }^{[20]}$. 海洋水体中, 可以 使用 PROFHMM 统计方法 [HMM ( Hidden Markov Models) 和 SOM ( Self-Organizing Topological Maps) 的组 合 ${ }^{[21]}$, 从海面数据中推断海洋生物地球化学变量垂直剖面; HMM 通过 SOM 来定义, SOM 被用来提供隐藏 的马尔科夫模型状态.

在湖泊水体中,水体垂向结构研究较少. 但实际上, 由于受风、浪等动力条件的影响, 湖泊藻颗粒垂向 分层呈现多种模式 ${ }^{[22]}$, 在藻颗粒生长的不同阶段 (休眠、复苏、生物量增加、上浮和积聚), 垂向分布有很大 变化 ${ }^{[22-23]}$; 特别在蓝藻水华形成阶段, 大量蓝藻颗粒漂浮于水面, 不能简单地用高斯模型模拟湖泊水体的藻 颗粒垂向分布结构, 需要分不同情形进行定量表达. Xue 等 ${ }^{[24]}$ 在巢湖的研究发现, 风速是引起藻颗粒短时间 内垂向迁移的重要因素. 当风速大于 $3 \sim 4 \mathrm{~m} / \mathrm{s}$ 时, 水柱内上下混合均匀, 藻颗粒呈现垂向均一分布. 在弱风 条件下 $(2 \sim 3 \mathrm{~m} / \mathrm{s})$, 蓝藻自身浮力使其在水柱内上浮, 形成 Chl. a 浓度最大值在水表面的高斯分布. 而在风 速很低或无风的情况下 $(<2 \mathrm{~m} / \mathrm{s})$, 藻颗粒大量聚集在水表面形成藻华, 此时 Chl. a 浓度垂向呈现指数或幂 指数分布. 据此把巢湖藻类垂向分布归结为 4 种类型 (图 1): (1) 垂向均一分布型; (2) 高斯分布型; (3) 指 数分布型; (4) 幂函数分布型. 同时给出了上述 4 种分布类型的数学描述函数 (表 1$)$.

\section{3 水下光场分布的定量表达与数值模拟}

光进人水体, 受水中粒子 (水分子、藻类等有机颗粒物、黄色物质、无机悬浮物等) 吸收和散射共同影响, 产生衰减, 但在不同波长, 衰减程度差别很大. 水下光场是对水体组分在特定太阳角度、风速、气象条件下 水体表观光学特性的综合反映, 主要由辐亮度、辐照度、漫衰减系数、平均余弦等表观光学量来反映水下光 场的分布情况. 

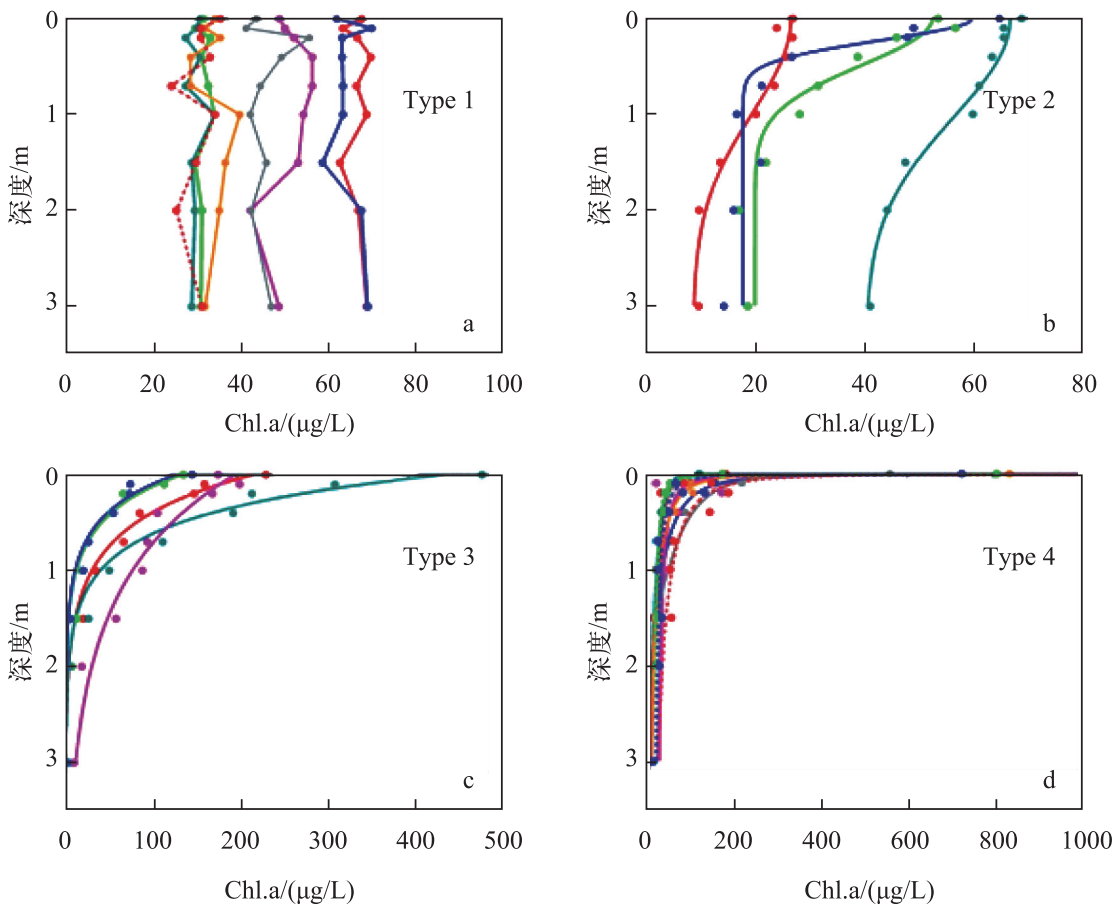

图 1 巢湖 Chl. a 浓度垂向分布类型图 (Type1:垂向均一,Type2: 高斯,Type3:指数,Type4:幕函数)

Fig.1 Chlorophyll-a concentration profiles with original data points and fitted curves for the four vertical chlorophyll-a profile classes: (a) Type 1, vertically uniform; (b) Type 2, Gaussian distribution;

(c) Type 3, exponential distribution; and (d) Type 4, negative power function distribution

$$
\mu \frac{\partial L(\tau ; \mu, \varphi)}{\partial \tau}=-L(\tau ; \mu, \varphi)+\omega_{0}(\tau) \iiint_{(\mu, \varphi) \in \Xi} L\left(\tau ; \mu^{\prime}, \varphi^{\prime}\right) \times \widetilde{\beta}\left(\tau ; \mu^{\prime}, \varphi^{\prime} \rightarrow \mu, \varphi\right) \mathrm{d} \mu^{\prime} \mathrm{d} \varphi^{\prime}+S(\tau ; \mu, \varphi)
$$

式中, $L(\tau ; \mu, \varphi)$ 为在光学深度 $\tau$ (光到达的最大深度)、方向 $(\mu, \varphi)$ 上的非偏振光谱辐照度; $\omega_{0}$ 为散射和衰 减的比值; $\tilde{\beta}$ 为散射相函数; $S$ 为水体内部光源.

藻颗粒垂直分层导致光在水体中的传播路径和方向发生变化, 是影响水下光场分布的重要原因 ${ }^{[8]}$. 在 垂直分层的水体中, 存在高吸收水层和高散射水层; 在高散射水层中, 水下光场的强度随光学深度减小得更 加缓慢;在高吸收的水层中,水下光场的强度 随光学深度减小得更加迅速 ${ }^{[25]}$. 在仅有吸 收、没有散射的介质中,光场的角度分布不会 随传输距离的增加而发生改变. 事实上,湖 泊水体具有高散射的特性 ${ }^{[26]}$, 水体中悬浮性 藻颗粒的存在使湖水的散射具有明显的前向 峰值 ${ }^{[27]}$. 因此, 光束在水体中传输时, 随传输 距离、散射次数的不同,光场的角度分布会有 明显的变化. 传输距离越长, 散射次数越多, 光场的漫射分布特征就越明显. 特别是在藻 华期间, 高度累积在水体表层的生物量, 使得 下层水体获取的有效光合作用辐射大幅衰 减,称为藻华过程的“自我遮蔽”效应 ${ }^{[28-29]}$,

表 1 巢湖 Chl. a 浓度垂向分布类型及其函数表达"

Tab. 1 Vertical distribution type and mathematic expression of chlorophyll-a concentration in Lake Chaohu, China

\begin{tabular}{lcll}
\hline \multicolumn{2}{c}{$\begin{array}{c}\text { Chl. a 浓度垂向 } \\
\text { 分布类型 }\end{array}$} & 函数表达 \\
\hline Type 1 & 垂向均一 & $f_{1}(z)=C$ \\
Type 2 & 高斯 & $f_{2}(z)=C_{0}+\frac{h}{\sigma \sqrt{2} \pi} \exp \left[-\frac{1}{2}\left(\frac{z}{\sigma}\right)^{2}\right]$ \\
Type 3 & 指数 & $f_{3}(z)=m_{1} \cdot \exp \left(m_{2} \cdot z\right)$ \\
Type 4 & 幂函数 & $f_{4}(z)=n_{1} \cdot z^{n_{2}}$ \\
\hline
\end{tabular}

$* C_{0} 、 h 、 \sigma 、 m_{1} 、 m_{2} 、 n_{1} 、 n_{2}$ 均为 Chl. $\mathrm{a}$ 浓度垂向分布的结构参 数, $z$ 为水深. 
造成了藻华期间水体真光层深度降低,下行辐照度减少.

水下光场的研究可以通过辐射传输理论模型或者野外实测的方式开展. 随着水体光辐射传输理论的 发展, 数值计算方法在水下光场模拟中得到了广泛应用, 根据上述辐射传输理论模型得到水下光场分布的 方法有:Monte Carlo 光线追踪法 ${ }^{[30-35]}$ 、不变嵌入法 ${ }^{[25]}$ 、离散坐标法 ${ }^{[36]}$ 、矩阵算法 ${ }^{[37-40]}$ 等; 其中, Mobley 利用不 变嵌人方法开发的 Hydrolight 商业软件 ${ }^{[41]}$ 成为开展水下光场模拟研究的主要技术手段. Hydrolight 可以模 拟不同水体状况、角度、波长下的水下光场,还可以考虑拉曼散射和苂光效应的影响,因此利用 Hydrolight 辐 射传输模拟技术可以得到相对准确的水下光场. Liu 等 ${ }^{[42-43]}$ 提出了一种快速准确模拟水下光场辐照度的模 型, 基于 Hydrolight 参数的敏感性分析, 建立了参数查找表, 平均误差在 $2 \%$ 左右. Hydrolight 假设水体水平均 一,是一维的,无法解决复杂边界诸如水华聚集到表面后有异于传统水体辐射过程的问题.

蒙特卡洛方法 (Monte Carlo, MC) 是利用随机数对随机过程进行数值模拟的方法, 可以模拟以任意数学 模型表述的光在介质中的辐射传输过程 ${ }^{[44]}$, 是目前水体光学模型中唯一能解决有复杂边界问题的方法. 此 外,该方法可以直观地表达辐射传输过程中光束被水中粒子 (水分子、悬浮颗粒物等) 吸收和散射过程,物理 结构清晰. 它可以对任意人射光线、散射相函数和固有光学属性分布情况下的水下光场进行三维模拟,可 以解决一维模型所不能解决的问题, 更加符合客观世界的实际情况. Monte Carlo 方法具有较强的灵活性, 可 以模拟野外测量难以达到的极限条件 ${ }^{[45]}$. 在水体光学研究领域, Plass 等 ${ }^{[30-35]}$ 已经用 Monte Carlo 方法解决 不同的海洋光学问题. 国内学者在模拟浮标浮体阴影及其安装的仪器自阴影对水下光辐射测量的影响 ${ }^{[46]}$ 、 理想简单条件下的水下光场 Monte Carlo 模拟及水下光场的二向反射特性研究 ${ }^{[45,47]}$ 方面取得了一定的成果. 上述分析表明, 基于能对任意用数学公式和离散数值给出的边界条件和参数分布进行模拟的特点, Monte Carlo 可用于藻颗粒富集、水体具有垂向分层、具有复杂边界条件的富营养化湖泊的水下光场模拟,但目前这 方面研究工作还没有开展. 但是, Monte Carlo 方法存在运行效率慢的问题, 在模拟大量光子减少噪声的同 时, 加剧了运行的负担. 即使有提高计算速度的方法 (如方差缩减技术等), 但这些方法只在一定程度上提 高光子的利用效率. Monte Carlo 方法的计算数据相对独立, 串行数据处理少等优良的并行化特性, 可以使用 并行化方法来提高运行效率, 可以达到几百上千的加速比 ${ }^{[48]}$.

\section{4 垂向异质水体遥感反射比的定量表达}

对 $\mathrm{I}$ 类水体而言, 结合水体的固有光学特性 (水体的总吸收 $a$ 和总后向散射 $b_{\mathrm{b}}$ ), 有 ${ }^{[49-50]}$ :

$$
R_{\mathrm{rs}}=\frac{f}{Q} \cdot \frac{b_{\mathrm{b}}}{a} \text { 或 } R=f \frac{b_{\mathrm{b}}}{a}
$$

式中, $f=0.975-0.629 \mu_{0}^{[51]}, \mu_{0}$ 为水下平均余弦; $Q$ 为上行辐照度与上行辐射亮度的比值; 一般地, $f / Q=$ $0.0945^{[52]} 、 0.0922^{[34,53]}$ 或 $0.0924^{[54]}$. 水体垂向光学均质时, $b_{\mathrm{b}}$ 和 $a$ 可以随水深而变化, 但 $b_{\mathrm{b}} / a$ 不变 ${ }^{[55]}$; 或 者 $b_{\mathrm{b}}$ 和 $a$ 都随叶绿素而协同变化 ${ }^{[27]}$, 此时水体的光学属性被浮游植物主导, $a(z)$ 和 $b_{\mathrm{b}}(z)$ 的垂直结构能够 通过同一个深度函数 $h(z)$ 来导出, 即 $a(z)=a(0) h(z), b_{\mathrm{b}}(z)=b_{\mathrm{b}}(0) h(z)$. 因此, 光学均质时, 在每个深度 $z$ 上,水体都有相同的反射率, 即 $R / f=\left(b_{b z} / a_{z}\right)$, 此时:

$$
\overline{\left(\frac{b_{\mathrm{b}}}{a}\right)}=\frac{\overline{b_{\mathrm{b}}}}{\overline{\bar{a}}}
$$

水体在垂向上是否光学均质, 可以通过光学均质指数 $H$ 来判断 ${ }^{[55]}$ :

$$
H=\overline{\left(\frac{b_{\mathrm{b}}}{a}\right)} / \overline{\frac{b_{\mathrm{b}}}{\bar{a}}}
$$

当水体光学垂向非均质时, 适用于光学均质水体的式 (3) 就会带来较大误差. Gordon 等 ${ }^{[56]}$ 认为如果光 活性物质 $C$ 具有垂直分布结构 $C(z)$ 时, 遥感反射比则等同于该光活性物质垂直均质 $\overline{C_{\mathrm{s}}}$ 时的情况:

$$
\overline{C_{\mathrm{s}}}=\frac{\int_{0}^{Z_{\infty}} C(z) g(z) \mathrm{d} z}{\int_{0}^{Z_{\infty}} g(z) \mathrm{d} z}
$$


式中, $z_{90}$ 为向下辐照度衰减到 $90 \%$ 的深度, $g(z)$ 权重函数为:

$$
g(z)=\exp \left[-2 \int_{0}^{z} K\left(z^{\prime}\right) \mathrm{d} z^{\prime}\right]
$$

式中, $K$ 为漫垂直衰减系数. 此时, 对于式 (3) 的遥感反射比而言, $b_{\mathrm{b}} / a$ 可用 $\overline{\left(\frac{b_{\mathrm{b}}}{a}\right)}$ 来表达 ${ }^{[56-57]}:$

$$
\overline{\left(\frac{b_{\mathrm{b}}}{a}\right)}=\frac{\int_{0}^{z_{\infty}} \frac{b_{\mathrm{b}}(z)}{a(z)} f(z) \mathrm{d} z}{\int_{0}^{z_{\infty}} f(z) \mathrm{d} z}
$$

Zaneveld $^{[58]}$ 从理论上导出了光学垂直结构变化情况下遥感反射比的关系表达; 然而, 该关系式由于包含 了体散射函数和辐亮度分布, 被认为过于复杂而难以应用 ${ }^{[55]}$. Gordon ${ }^{[27]}$ 对这个权重函数所隐藏的假设条件 ( 即分层海洋水体的反射率等同于浮游植物色素浓度均质的海洋水体的反射率, 这个浮游植物色素浓度是 随水深变化的函数的平均值), 使用 Monte Carlo 模拟实验, 从理论上加以证明. 同样使用 Monte Carlo 模型, Piskozub 等 ${ }^{[57]}$ 表明式 (7) 所示的权重函数过分依赖表层水体的光学特性, 即会赋予表层水体更多的权重. 于是又发展了另外一种权重函数 $f(z)$ :

$$
f(z)=\frac{\mathrm{d}}{\mathrm{d} z} \exp \left[-\int_{0}^{z} 2 K\left(z^{\prime}\right) \mathrm{d} z^{\prime}\right]
$$

于是有 ${ }^{[55]}$ :

$$
\overline{\left(\frac{b_{\mathrm{b}}}{a}\right)}=\int_{0}^{\infty} \frac{b_{\mathrm{b}}(z)}{a(z)} f(z) \mathrm{d} z
$$

$f(z)$ 是 $g(z)$ 的一阶导数, 在水体光活动层的顶部具有最大值, 不像 $g(z)$, 它不是单调递减函数. 但二者 都涉及到 $2 K$ 的计算, 不同的情况, 可选择不同的计算方式: $2 K_{\mathrm{d}} 、 K_{\mathrm{u}}+K_{\mathrm{d}} 、 K_{\mathrm{d}}+c$ ( $c$ 为漫衰减系数). 对于光学遥 感而言,一般情况下 ${ }^{[57]}$, 用 $2 K_{\mathrm{d}}$ 的方法优于用 $K_{\mathrm{u}}+K_{\mathrm{d}}$ 的方法, $K_{\mathrm{u}}+K_{\mathrm{d}}$ 的计算结果要好于 $K_{\mathrm{d}}+c$. 另外, 通过辐照 度剖面计算漫衰减系数, 要好于通过固有光学特性的计算 ${ }^{[55,57]}$.

在悬浮颗粒垂直平均的反演时, Pitarch 等 ${ }^{[59]}$ 给出了一个权重函数, 即:

$$
g(z, \lambda)=\exp \left\{-\int_{z}^{0}\left[K_{\mathrm{d}}\left(z^{\prime}, \lambda\right)+K_{\mathrm{u}}\left(z^{\prime}, \lambda\right)\right] \mathrm{d} z^{\prime}\right\}
$$

如果输出深度不是很密, Ecolight 能够提供不很准确的 $K_{\mathrm{u}}$ 和 $K_{\mathrm{d}}$, Ecolight 能够自动增加靠近输出深度的 新深度. 因此, 通过每个点的 Ecolight 模拟, 可以获得 $K_{\mathrm{u}}$ 和 $K_{\mathrm{d}}$. 与 Hydrolight 相比, Ecolight 使用同样的输人 数据. 公式 (11) 的权重函数依赖波长, 为了获得单一波长处的平均值, 需要按照公式 (12) 进行波长平均:

$$
\langle s m\rangle=\frac{1}{N_{\lambda}} \sum_{j=1}^{N_{4}} \frac{\int_{-\infty}^{0} s m(z) g\left(z, \lambda_{j}\right) \mathrm{d} z}{\int_{-\infty}^{0} g\left(z, \lambda_{j}\right) \mathrm{d} z}
$$

\section{5 藻类垂向异质对水色参数遥感反演模型的影响}

目前, 生物光学模型和水体光学参数的测量和使用大都假设水体垂向均一, 但是不考虑水体光学组分 的垂直非均匀分布会带来很大误差 ${ }^{[12,60-61]}$. Sathyendranath 等 ${ }^{[12]}$ 认为, 在不考虑叶绿素浓度垂直分布情况下 计算光学深度和真光层总叶绿素浓度, 最大相对误差可能超过 100\%. I 类水体叶绿素垂向一般呈高斯分 布, 如果不考虑其垂向结构, 对遥感反射比造成的误差最低大于 $5 \%$, 极端条件下可能超过 $70 \%$, 近表层水体 的衰减系数越小, 下层水体对遥感反射比的影响越强 ${ }^{[62-63]}$.

与藻类垂向均一相比, 藻类垂向非均匀分布主要影响遥感反射比的大小及光谱形 ${ }^{\left[{ }^{[64]}\right.}$. 在 I 类水体中 叶绿素浓度垂向呈高斯分布时, 叶绿素浓度的高斯峰值趋向于增加绿光波段的遥感反射比, 减少蓝光波段 的遥感反射比 ${ }^{[13]}$. 赤潮多发区, 垂向非均匀情况下的遥感反射比要高于垂向均匀的情况 (藻总量相同), 叶 绿素浓度非均匀剖面参数的变化主要影响遥感反射比的绿黄波段, 对蓝、红波段几乎没有影响 ${ }^{[65]}$. 马孟臬 
等 ${ }^{[66]}$ 利用 Hydrolight 模拟藻类多种高斯垂向分布条件下,藻类垂向分布对典型叶绿素 a 反演算法波段比值 法的影响. 结果表明,藻类垂向非均匀分布尤其是水下 $0.5 \mathrm{~m}$ 范围内,将会严重干扰波段比值算法的准确 性, 导致反演算法的失效. 藻类垂向异质对 $R_{\mathrm{rs}}$ 的影响程度受水体光学活性物质浓度、垂向结构、水体衰减系 数等的共同影响. 已有的水质参数反演算法大多基于表层几十公分的混合样或者单一水层建立的模型, 当 藻类垂向异质对 $R_{\mathrm{rs}}$ 的影响很大时, 势必会将这种误差引人水质参数反演模型, 引起反演产品的高估或低估, 这对正确估计水质参数浓度的空间分布会产生干扰,而且这种干扰可能是空间不均匀分布的.

\section{6 展望}

太湖等东部地区湖泊是我国最为典型的富营养化湖泊之一, 近年来以蓝藻为优势类群 ${ }^{[67-68]}$ 的藻华暴发 频繁 ${ }^{[69-70]}$, 藻颗粒或大量漂浮于水面或淹没于水体. 这些在水平与垂直方向异质分布的藻颗粒, 改变了传 统的水色遥感理论基于水体水平和垂向均一的假设前提, 水色参数遥感定量反演的不确定大大增加. 因 此, 藻颗粒及其引起的水体垂向结构变化, 成为水色遥感, 特别是富营养化湖泊水色遥感不可忽略的问题. 目前主要存在 3 个方面还不清楚, 亟需加强研究:

1 ) 水面藻颗粒密度与遥感反射比的定量关系不清. 遥感反射比除了受水体不同组分的影响外, 还受 水一气界面的影响. 藻颗粒在不同水文气象条件下的富集, 改变了水面的物质组成, 水体的透光性下降,在 极端情况下甚至使得自然光难以穿透这些大量积聚而漂浮在水面的藻颗粒, 从而改变了水体的辐射传输过 程. 因此, 水面藻颗粒密度显著影响着水-气界面光的透射率, 改变了透过水面进人水体的光通量, 直接影 响着水体的遥感反射比. 遥感反射比的细微变化, 包含了藻颗粒在水面的富集信息, 反映了水面不同的藻 颗粒密度;然而, 目前尚不清楚不同的水面藻颗粒密度与遥感反射比的响应关系.

2) 水中藻颗粒对水下光场结构的影响机制不清. 受水文气象条件的影响以及藻颗粒自身特性的限制, 水体中的藻颗粒存在着垂向分层现象, 也改变着自然光的辐射传输路径和方向,一定程度上影响着水下光 场结构, 进而影响着水体的遥感反射比. 实际上, 藻颗粒的垂向分层具有不同的垂直梯度和结构模式. 不同 的梯度与结构, 对水下光场的影响不同,但影响机制目前尚不清楚.

3 ) 藻颗粒本身的光学特性需要进一步明确. 遥感反射比取决于水体的固有光学特性, 固有光学特性独 立于介质的环境光场, 由介质本身决定. 富营养化湖泊中, 藻颗粒是最重要的一种介质, 它们或以单一藻颗 粒、或以颗粒集聚体的形式存在, 因此具有不同的粒径大小、形状结构和色素组成, 决定着不同的固有光学 特性( 吸收和散射特性), 对遥感反射比有着不同程度的影响.

藻颗粒在水面和水体中的大量富集, 引起水面物质组成、水体光学特性以及水体辐射传输过程的变化, 是富营养化湖泊水色遥感研究中面临的基本理论问题. 对这些问题的研究, 不但可以补充完善湖泊水色遥 感的基本理论和方法, 而且能够推动湖泊水色遥感和湖泊定量遥感的发展, 具有较大的科学意义. 另外, 通 过藻颗粒及其垂向不均匀下的非传统水色遥感研究, 可以定量揭示遥感反射比与水柱中藻颗粒密度的定量 关系, 有助于实现藻总量的精确遥感反演与估算, 为藻类生态灾害监测和预测预警服务, 具有较大的应用价 值和现实意义.

\section{7 参考文献}

[1] 马荣华, 唐军武, 段洪涛等. 湖泊水色遥感研究进展. 湖泊科学, 2009, 21 (2): 143-158. DOI 10. $18307 / 2009.0201$.

[ 2 ] Blasco D. Observations on the diel migration of marine dinoflagellates off the Baja California coast. Marine Biology, 1978, 46: $41-47$.

[ 3 ] Renger GM, Völker M, Eckert HJ et al. On the mechanism of Photosystem II deterioration by UV-B irradiation. Photochemistry and Photobiology, 1989, 49: 97-105.

[ 4 ] Sherman B, Webster IT. A model for the light-limited growth of buoyant phytoplankton in a shallow turbid water body. Marine and Freshwater Research, 1994, 45: 847-862.

[ 5 ] Villarino ML, Figueiras FG, Jones KJ et al. Evidence of in situ diel vertical migration of a red-tide microplankton species in Ria de Vigo (NW Spain). Marine Biology, 1995, 123: 607-617. 
[ 6 ] Jordan BR. The effects of ultraviolet-B radiation on plants: a molecular perspective. Advances in Botanical Research, 1996, 22: $97-162$.

[ 7 ] Whittington J, Sherman B, Green D et al. Growth of Ceratium hirundinella in a subtropical Australian reservoir: the role of vertical migration. Journal of Plankton Research, 2000, 22: 1025-1045.

[ 8 ] Lewis MR, Cullen JJ, Platt T. Phytoplankton and thermal structure in the upper ocean: consequence of nonuniformity in chlorophyll profile. Journal of Geophysical Research, 1983, 88: 2565-2570.

[ 9 ] Uitz J, Claustre H, Morel A et al. Vertical distribution of phytoplankton communities in open ocean: An assessment based on surface chlorophyll. Journal of Geophysical Research: Oceans (1978-2012), 2006, 111(C8).

[10] 胡 毅, 陈 坚. 夏季台湾浅滩周边海域叶绿素 a 苂光垂直分布对温盐的响应分析. 热带海洋学报, 2008, 27 (2) : 18-24.

[11] Nyoman D, Kanno S, Arthana I. Study on the vertical distribution of chlorophyll in coastal ocean; development of vertical model function at western Sumbawa sea. Ecotrophic, Journal of Environmental Science, 2006, 1(2) : 1-9.

[12] Sathyendranath S, Platt T. Remote sensing of ocean chlorophyll: consequence of nonuniform pigment profile. Applied Optics, 1989, 28: 490-495.

[13] 席 颖, 杜克平, Lee Z 等. 叶绿素浓度垂直不均一分布对于分层水体表观光学特性的影响. 光谱学与光谱分 析, 2010, 30(2): 489-494.

[14] 曹文熙. 叶绿素垂直分布结构对离水辐亮度光谱特性的影响．海洋通报, 2000, 19(3): 30-37.

[15] Millán-Núñez R, Alvarez-Borrego S, Trees C. Modeling the vertical distribution of chlorophyll in the California current system. Journal of Geophysical Research, 1997, 102( C4) : 8587-8595.

[16] Hidalgo-Gonzalez RM, Alvarez-Borrego S. Chlorophyll profiles and the water column structure in the gulf of california. Oceanologica Acta, 2001, 24: 19-28.

[17] 冯春晶. 基于人工神经网络的海中叶绿素浓度垂直分布特征研究 [学位论文]. 青岛: 中国海洋大学, 2004.

[18] 刘 斌. 一类水域中从海面反射比反演叶绿素浓度垂直分布初探 [学位论文]. 青岛: 中国海洋大学, 2009.

[19] Frettẽ Ø, Erga SR, Stamnes JJ et al. Optical remote sensing of waters with vertical structure. Applied Optics, 2001, 40: 1478-1487.

[20] Yang Q, Stramski D, He MX. Modeling the effects of near-surface plumes of suspended particulate matter on remote-sensing reflectance of coastal waters. Applied Optics, 2013, 52: 359-374.

[21] Charantonis AA, Badran F, Thiria S. Retrieving the evolution of vertical profiles of chloropyll-a from satellite observations using Hidden Markov Models and Self-Organizing Topological Maps. Remote Sensing of Environment, 2015, 163: 229-239.

[22] Cao HS, Kong FX, Luo LC et al. Effects of wind and wind-induced waves on vertical phytoplankton distribution and surface blooms of Microcystis aeruginosa in Lake Taihu. Journal of Freshwater Ecology, 2006, 21: 231-238.

[23] 孔繁翔, 高 光. 大型浅水富营养化湖泊中蓝藻水华形成机理的思考. 生态学报, 2005, 25(3): 589-595.

[24] Xue K, Zhang Y, Duan H et al. A remote sensing approach to estimate vertical profile classes of phytoplankton in a eutrophic lake. Remote Sensing, 2015, 7(11) : 14403-14427.

[25] Mobley CD. Light and water: Radiative transfer in natural waters. New York: Academic Press, 1994.

[26] Ma R, Pan D, Duan H et al. Absorption and scattering properties of water body in Taihu Lake, China: backscattering. International Journal of Remote Sensing, 2009, 30: 2321-2335.

[27] Gordon HR. Diffuse reflectance of the ocean: influence of nonuniform phytoplankton pigment profile. Applied Optics, 1992, 31: 2116-2129.

[28] Susanne K. Assessing secchi and photic zone depth in the Baltic Sea from satellite data. Remote Sensing of Environment, 2003, 32 : 577-585.

[29] Boyd PW, Andrew J, Watson J et al. A mesoscale phytoplankton bloom in the polar Southern Ocean stimulated by iron fertilization. Nature, 2000, 407: 695-702.

[30] Plass GN, Kattawar GW. Monte carlo calculations of light scattering from clouds. Applied Optics, 1968, 7: 415-419.

[31] Plass GN, Kattawar GW. Radiative transfer in an atmosphere-ocean system. Applied Optics, 1969, 8: 455-466.

[32] Kirk JTO. Monte Carlo study of the nature of the underwater light field in, and the relationships between optical properties of, turbid yellow waters. Marine and Freshwater Research, 1981, 32: 517-532.

[33] Gordon HR. Ship perturbation of irradiance measurements at sea. 1: Monte Carlo simulations. Applied Optics, 1985, 24 : 
4172-4182.

[34] Morel A, Gentili B. Diffuse reflectance of oceanic waters: Its dependence on sun angle as influenced by the molecular scattering contribution. Applied Optics, 1991, 30: 4427-4438.

[35] Mobley CD, Sundman LK. Effects of optically shallow bottoms on upwelling radiances: Inhomogeneous and sloping bottoms. Limnology and Oceanography, 2003, 48: 329-336.

[36] Jin Z, Stamnes K. Radiative transfer in nonuniformly refracting layered media: Atmosphere-ocean system. Applied Optics, 1994, 33: 431-442.

[37] 张 鉴, 何晓雄, 赵凤生. 利用大气-海洋系统辐射传输模拟水色遥感信息量的变化特性. 量子电子学报, 2003, $20(5)$ : 623-628.

[38] 何贤强, 潘德炉, 白 雁等. 基于矩阵算法的海洋-大气耦合矢量辐射传输数值计算模型. 中国科学: D 辑: 地球 科学, 2006, 36(9) : 860-870.

[39] 何贤强, 潘德炉, 白 雁等. 基于辐射传输数值模型 PCOART 的大气漫射透过率精确计算. 红外与毫米波学报, $2008,27(4)$ : 303-307.

[40] 何贤强, 潘德炉, 白 雁等. 海洋-大气耦合矢量辐射传输粗糙海面模型. 光学学报, 2010, 30(3): 618-624.

[41 ] Mobley CD, GentiliB, Gordon HR et al. Comparison of numerical models for computing underwater light fields. Applied Optics, 1993,32 : 7484-7504.

[42] Liu CC, Woods JD, Mobley CD. Optical model for use in oceanic ecosystem models. Applied Optics, 1999, 38: 4475-4485.

[43] Liu CC, Carder KL, Miller RL et al. Fast and accurate model of underwater scalar irradiance. Applied Optics, 2002 , 41: 4962-4974.

[44] 弓洁琼, 詹海刚, 刘大召. 遥感遥测中偏振信息的研究进展. 光谱学与光谱分析, 2010, 30(4) : 1088-1095.

[45] 凌在盈. 基于 Monte Carlo 方法的水体二向反射分布函数 (brdf) 模拟 [学位论文]. 杭州: 浙江大学, 2010.

[46] 曹文熙, 吴廷芳, 杨跃忠等. 光学浮标阴影效应的蒙特卡洛模拟. 高技术通讯, 2003，(3)：80-84.

[47] 唐军武, 田国良, 陈清莲. 离水辐射非朗伯特性的 Monte Carlo 模拟及分析. 海洋学报, 2000, (2): 48-57.

[48] 杜克平, 薛 坤. GPU 加速的水体辐射传输 Monte Carlo 模拟模型研究. 湖泊科学, 2016, 28(3).

[49] Morel A, Prieur L. Analysis of variations in ocean color. Limnology and Oceanography, 1977, 22: 709-722.

[50] Gordon HR, Brown OB, Jacobs MM. Computed relationships between the inherent and apparent optical properties of a flat homogeneous ocean. Applied Optics, 1975, 14: 417-427.

[51] Kirk JTO. Dependence of relationship between inherent and apparent optical properties of water on solar altitude. Limnology and Oceanography, 1984, 29: 350-356.

[52] Gordon HR, Brown OB, Evans RH et al. A semianalytic radiance model of ocean color. Journal of Geophysical Research, 1988, 93( D9) : 10909-10924.

[53] Lee Z, Carder KL, Mobley CD et al. Hyperspectral remote sensing for shallow waters I: A semianalytical model. Applied Optics, 1998, 37: 6329-6338.

[54] Ma R, Tang J, Dai J. Bio-optical model with optimal parameter suitable for Taihu Lake in water colour remote sensing. International Journal of Remote Sensing, 2006, 27: 4305-4328.

[55] Zaneveld JR, Barnard AH, Boss E. Theoretical derivation of the depth average of remotely sensed optical parameters. Optics Express, 2005, 13: 9052-9061.

[56] Gordon HR, Clark DK. Remote sensing optical properties of a stratified ocean: an improved interpretation. Applied Optics, 1980, 19: 3428-3430.

[57] Piskozub J, Neumann T, Wozniak L. Ocean color remote sensing: Choosing the correct depth weighting function. Optics Express, 2008, 16: 14683-14688.

[58] Zaneveld JR. Remotely sensed reflectance and its dependence on vertical structure: a theoretical derivation. Applied Optics, 1982, 21: 4146-4150.

[59] Pitarch J, Odermatt D, Kawka M et al. Retrieval of particle scattering coefficients and concentrations by genetic algorithms in stratified lake water. Remote Sensing, 2014, 6(10): 9530-9551.

[60] Nanu L, Robertson C. The effect of suspended sediment depth distribution on coastal water spectral reflectance: Theoretical simulation. International Journal of Remote Sensing, 1993, 14: 225-239. 
[61] Ballestero D. Remote sensing of vertically structured phytoplankton pigments. Top Meteor Oceanography, 1999, 6: 14-23.

[62] Stramska M, Stramski D. Effects of a nonuniform vertical profile of chlorophyll concentration on remote-sensing reflectance of the ocean. Applied Optics, 2005, 44: 1735-1747.

[63] Xiu P, Liu Y, Tang J. Variations of ocean colour parameters with nonuniform vertical profiles of chlorophyll concentration. International Journal of Remote Sensing, 2008, 29: 831-849.

[64] Kutser T, Metsamaa L, Dekker AG. Influence of the vertical distribution of cyanobacteria in the water column on the remote sensing signal. Estuarine, Coastal and Shelf Science, 2008, 78: 649-654.

[65] 王云飞, 贺明霞. 东海赤潮多发区非均匀叶绿素浓度剖面对遥感反射比的影响. 中国海洋大学学报: 自然科学 版, 2010, 40(10): 100-104.

[66] 马孟香, 张玉超, 钱 新等. 藻类垂向分布对内陆湖泊叶绿素 a 反演算法影响的模拟研究. 红外与毫米波学报, 2014, 33(6) : 666-673.

[67] 成 芳. 太湖水体富营养化与水生生物群落结构的研究 [学位论文]. 苏州: 苏州大学, 2010.

[68] Chen Y, Qin B, Teubner K et al. Dokulil. Long-term dynamics of phytoplankton assemblages: Microcystis domination in Lake Taihu, a large shallow lake in China. Journal of Plankton Research, 2003, 25: 445-453.

[69] 马荣华, 孔繁翔, 段洪涛等. 基于卫星遥感的太湖蓝藻水华时空分布规律认识. 湖泊科学, 2008, 20(6): 687694. DOI 10. 18307/2008. 0605.

[70] Duan H, Ma R, Xu X et al. Two-decade reconstruction of algal blooms in China's Lake Taihu. Environmental Science and Technology, 2009, 43: 3522-3528. 